\title{
ANALYSIS OF BEAMS WITH TRANSVERSAL GRADATIONS OF THE YOUNG'S MODULUS AND VARIABLE DEPTHS BY THE MESHLESS METHOD
}

\author{
Ladislav SÁTOR ${ }^{1}$, Vladimír SLÁDEK ${ }^{1}$, Ján SLÁDEK
}

\begin{abstract}
A numerical analysis based on the meshless local PetrovGalerkin (MLPG) method is proposed for a functionally graded material FGM (FGMfunctionally graded material) beam. The planar bending of the beam is considered with a transversal gradation of Young's modulus and a variable depth of the beam. The collocation formulation is constructed from the equilibrium equations for the mechanical fields. Dirac's delta function is employed as a test function in the derivation of a strong formulation. The Moving Least Squares (MLS) approximation technique is applied for an approximation of the spatial variations of all the physical quantities. An investigation of the accuracy, the convergence of the accuracy, the computational efficiency and the effect of the level of the gradation of Young's modulus on the behaviour of coupled mechanical fields is presented in various boundary value problems for a rectangular beam with a functionally graded Young's modulus.
\end{abstract}

Address

1 Department of Mechanics, Institute of Constrution and Architecture, Slovak Academy of Sciences, Dúbravská cesta 9, 84503 Bratislava 45, Slovakia

Corresponding author: ladislav.sator@savba.sk

\section{Keywords}

- Planar bending,

- FGM beam

- Moving Least Squares approximation scheme,

- meshless local Petrov -Galerkin (MLPG) method,

- collocation formulation.

\section{INTRODUCTION}

In conventional laminated composite structures, homogeneous elastic laminae are bonded together to obtain enhanced mechanical properties. However, the abrupt change in material properties across the interface between different materials can result in large stress discontinuities leading to delamination. This undesirable effect can be eliminated by replacing the laminated composite structure with the functionally graded materials (FGM) structure, where the stress distributions are smooth.

FGM can be characterized as multiphase composites with phase volume fractions gradually varying in space in a pre-determined profile (Suresh and Mortensen, 1998; Koizumi et al., 1993; Yamanouchi, 1990). Plasma spraying, powder metallurgy and other technologies are used for the fabrication of FGM. In recent decades several approaches have been developed for modelling FGMs, such as the Mori-Tanaka scheme (Benveniste, 1987; Mori and Tanaka, 1973), a composite cylindrical assemblage model (Hashin, 1964, 1979), the simplified strength of materials method (Chamis and Sendeckyj, 1968; Gibson, 1994), etc. From a macroscopic point of view, an FGM is mostly isotropic at each material point, but the material properties can vary continuously or discontinuously in one, two or three directions.

The Euler-Bernoulli theory (EBT) of beam bending covers the case for small deflections of a beam which is only subjected to lateral loads. Note that in the EBT for homogeneous beams, the deflection of the beam is not coupled with the axial displacements, but the gradation of the Young's modulus across the beam's depth gives rise to a coupling between the beam deflection and axial deformations, even in the case of the pure transversal loading of the plate. Note 
that the beam bending problem can also be solved separately from the axial deformations in the case of the transversal gradation of the Young's modulus with the bending stiffness being modified not only because of the functional dependence of Young's modulus, but also owing to the coupling effect. In any case the governing equation for beam bending in the EBT is the ordinary differential equation (ODE) of the $4^{\text {th }}$, order either with variable or constant coefficients. The boundary conditions are expressed in terms of the deflection and its derivatives, which could also be of the $3^{\text {rd }}$ order. Recall that high order derivatives are inappropriate from the point of view of numerical methods because of the increasing inaccuracy when increasing their order.

In this paper, we pay attention to decreasing the order of the derivatives involved in the governing equation and the boundary conditions of the beam bending problems in the EBT, for the purpose of decreasing inaccuracies in the numerical computations. The decom- posed formulation is proposed by introducing a new field variable in addition to the deflection field. Then, the governing equations are of the $2^{\text {nd }}$ order ODE. The numerical solution developed for this FGM beam-bending problem is based on the meshless approximation of field variables and a strong formulation of the governing equations (collocation of ODE), which belongs to the class of Meshless Local Petrov-Galerkin (MLPG) methods (Atluri, 2004). The prescribed boundary conditions are also considered in a strong form by their collocation at the boundary nodes. The Moving Least Squares (MLS) approximation technique (Lancaster and Salkauskas, 1981) is used for approximating the primary field variables.

Several illustrative examples are considered for testing the accuracy, the convergence of the accuracy and the computational efficiency. The influence of gradation parameters on the response of the beam to static uniform loading is investigated and presented.

\section{MATHEMATICAL FORMULATION}

\subsection{Material properties}

Let us consider a functionally graded beam with a rectangular cross-section area, with a length of $L\left(x_{1} \in[0, L]\right)$, a depth of $h\left(x_{3} \in[-h / 2, h / 2]\right)$, and a width of $b\left(x_{2} \in[-b / 2, b / 2]\right)$, respectively, and the external loading is applied in the direction of the $\left(-x_{3}\right)$-axis. The material properties are graded continuously across the beam's depth. The transversal gradation of Young's modulus is assumed in the following form:

$$
E\left(x_{3}\right)=E_{0}\left[1+\zeta\left(\frac{1}{2} \pm \frac{x_{3}}{h}\right)^{p}\right] ; \quad \zeta=\frac{E( \pm h / 2)}{E_{0}}-1, E_{0}=E(\mp h / 2),
$$

where $\zeta$ and $p$ are the values of the level and exponent of the power-law gradation, respectively; $x_{3}$ is the lateral coordinate in the transversal direction being positively upward.

\subsection{Governing equations}

Based on the Euler-Bernoulli theory of planar bending, the normal stress on the cross-section is given by:

$$
\sigma_{11}\left(x_{1}, x_{3}\right)=E\left[u_{, 1}\left(x_{1}\right)-x_{3} w_{, 11}\left(x_{1}\right)\right]
$$

where $x_{1}$ is the axial coordinate taken along the geometrically central axis; $u\left(x_{1}\right)$ is the axial displacement at an arbitrary point on the beam; and $w\left(x_{l}\right)$ is the deflection. Assuming the dimensions in the cross-section ( $h$ and $b$ ) to be much smaller than $L$, we can consider the balance equations in an averaged sense, i.e., in a semi-integral form by taking the integrals of the forces and force moments over the cross-section of the beam.

If we assume the planar bending of the FGM beam from the equilibrium of the forces and moments of forces, the governing equations are given as:

$$
T_{i 1,1}+P_{i}=0, \quad M_{\beta, 1}-\varepsilon_{\beta \alpha 1} T_{1 \alpha}=0
$$

in which $T_{i j}:=\int_{S} \sigma_{i j} d S, \quad P_{i}:=\int_{C} \sigma_{i \alpha} n_{\alpha} d l, \quad M_{\beta}:=\varepsilon_{\beta \alpha 1} \int_{S} x_{\alpha} \sigma_{11} d S$, with $C$ being the oriented contour around $S ; \varepsilon_{\alpha \beta 1}$ stand for the components of a completely antisymmetric tensor $\left(\varepsilon_{231}=1\right)$; and $\beta=\stackrel{S}{2}, 3$. Obviously, $T_{i j}, M_{\beta}$ and $P_{j}$ stand for the integral of the stresses, the moments of stresses $\sigma_{11}$ over the cross-section, and the integral of the tractions along contour $C$, respectively.

Hence,

$$
\begin{aligned}
& M_{2,11}+P_{3}=0, \\
& T_{11,1}=0,
\end{aligned}
$$

where $M_{2}$ is the bending moment expressed as

$$
M_{2}\left(x_{1}\right)=\varepsilon_{231} \int_{S} x_{3} \sigma_{11}\left(x_{1}, x_{3}\right) d S=E_{0} b \int_{-h / 2}^{h / 2} x_{3}\left[1+\zeta\left(\frac{1}{2} \pm \frac{x_{3}}{h}\right)^{p}\right]\left[u_{, 1}\left(x_{1}\right)-x_{3} w_{, 11}\left(x_{1}\right)\right] d x_{3}=\frac{E_{0} b h^{2}}{12}\left\{ \pm 12 c \gamma_{p} u_{, 1}\left(x_{1}\right)-h \beta_{p} w_{, 11}\left(x_{1}\right)\right\}
$$


and the averaged axial stresses $T_{11}$ are given by

$$
T_{11}\left(x_{1}\right)=\int_{S} \sigma_{11}\left(x_{1}, x_{3}\right) d S=E_{0} b \int_{-h / 2}^{h / 2}\left[1+\zeta\left(\frac{1}{2} \pm \frac{x_{3}}{h}\right)^{p}\right]\left[u_{, 1}\left(x_{1}\right)-x_{3} w_{, 11}\left(x_{1}\right)\right] d x_{3}=E_{0} b\left\{h \omega_{p} u_{, 1}\left(x_{1}\right) \mp h^{2} c \gamma_{p} w_{, 11}\left(x_{1}\right)\right\} ;
$$

with $\gamma_{p}:=\zeta s_{p}, \quad \beta_{p}:=1+\zeta f_{p}, \quad \omega_{p}:=1+\frac{\zeta}{p+1}, \quad s_{p}:=\frac{1}{p+2}-\frac{1}{2(p+1)}, f_{p}:=\frac{12}{p+3}-\frac{12}{p+2}+\frac{3}{p+1}$,

and the factor $c=1$ is introduced formally as will be seen later.

The transversal load $P_{3}$ is given as

$$
P_{3}=\int_{b / 2}^{-b / 2} \sigma_{33} d x_{2}=-q \int_{b / 2}^{-b / 2} d x_{2}=q b
$$

Remark: Note that Eqs. (6) and (7) are reduced to the familiar expressions

$$
M_{2}\left(x_{1}\right)=\frac{E_{0} b h^{3}}{12} w_{, 11}\left(x_{1}\right)=-b \int_{-h / 2}^{h / 2} E_{0} x_{3}^{2} w_{, 11}\left(x_{1}\right) d x_{3}, \quad T_{11}\left(x_{1}\right)=E_{0} b h u_{, 1}\left(x_{1}\right)=b \int_{-h / 2}^{h / 2} E_{0} u_{, 1}\left(x_{1}\right) d x_{3},
$$

if the cross-section of the beam is homogeneous $\left(E=\right.$ const $=E_{0}$ or $\left.\zeta=0\right)$. The generalization of these relationships via replacing $E_{0}$ behind the integral signs by $E\left(x_{3}\right)$, which was given by Eq. (1), was an incorrect consideration of the functionally graded Young's modulus, since the results obtained

$$
M_{2}\left(x_{1}\right)=-\frac{E_{0} b h^{3}}{12} \beta_{p} w_{, 11}\left(x_{1}\right), \quad T_{11}\left(x_{1}\right)=E_{0} b h \omega_{p} u_{1}\left(x_{1}\right)
$$

would not include coupling between the deflection and axial deformations. Since these expressions can be obtained from (6) and (7) by taking $c=0$, the choice of the factor $c=1$ or $c=0$ can be used for switching on/off the mentioned coupling, respectively.

Let us consider the power-law gradation of the depth of the beam as

$$
h\left(x_{1}\right)=h_{0} h^{*}\left(x_{1}\right), \quad h^{*}\left(x_{1}\right)=1+\kappa\left(\frac{x_{1}}{L}\right)^{S}, \quad x_{1} \in[0, L] .
$$

Inserting (7) into (5) and (6) into (4), one can derive the governing equations for the deflection and axial displacement of the beam. For this purpose, it seems to be convenient to eliminate $u_{, 1}\left(x_{1}\right)$ from (6) by using

$$
E_{0} b h^{2}\left(x_{1}\right) u_{, 1}\left(x_{1}\right)=\frac{h\left(x_{1}\right) T_{11}\left(x_{1}\right)}{\omega_{p}} \pm c \frac{\gamma_{p}}{\omega_{p}} E_{0} b h^{3}\left(x_{1}\right) w_{, 11}\left(x_{1}\right)
$$

resulting from (7). Thus, from (6) and (10), we obtain

$$
M_{2}\left(x_{1}\right)=-D\left(x_{1}\right) w_{, 11}\left(x_{1}\right) \pm c \frac{\gamma_{p}}{\omega_{p}} h\left(x_{1}\right) T_{11}\left(x_{1}\right),
$$

in which

$$
D\left(x_{1}\right):=D_{0} D_{V}^{*} D_{H}^{*}\left(x_{1}\right), \quad D_{0}:=\frac{E_{0} h_{0}^{3} b}{12}, \quad D_{V}^{*}:=\beta_{p}-12 \frac{\left(c \gamma_{p}\right)^{2}}{\omega_{p}}, \quad D_{H}^{*}\left(x_{1}\right):=h^{* 3}\left(x_{1}\right)
$$

stand for the bending stiffness of the beam considered, the bending stiffness of the homogeneous beam with a constant depth, the correction factor due to the vertical gradation of Young's modulus $\left(D_{V}^{*}=1\right.$ if $\left.\zeta=0\right)$, and the correction factor due to the variable depth $\left(D_{H}^{*}\left(x_{1}\right)=1\right.$ if $\left.h^{*}=1\right)$, respectively.

Now, in view of (4), (8), and (11), we obtain

$$
D_{0} D_{V}^{*}\left(\frac{\partial^{2} D_{H}^{*}}{\partial x_{1}^{2}} w_{, 11}+2 \frac{\partial D_{H}^{*}}{\partial x_{1}} w_{, 111}+D_{H}^{*} w_{, 111}\right) \mp c \frac{\gamma_{p}}{\omega_{p}} h_{0} \frac{\partial^{2} h^{*}}{\partial x_{1}^{2}} T_{11}=q b
$$

Recall that $T_{11,1}$ and $T_{11,11}$ disappear in (12) because of Eq. (5). Inserting (7) into (13), we have

$$
D_{0} D_{V}^{*}\left(\frac{\partial^{2} D_{H}^{*}}{\partial x_{1}^{2}} w_{, 11}+2 \frac{\partial D_{H}^{*}}{\partial x_{1}} w_{, 111}+D_{H}^{*} w_{, 111}\right)+\frac{E_{0} h_{0}^{3} b\left(c \gamma_{p}\right)^{2}}{\omega_{p}} h^{* 2} \frac{\partial^{2} h^{*}}{\partial x_{1}^{2}} w_{, 11} \mp c E_{0} h_{0}^{2} b \gamma_{p} h^{*} \frac{\partial^{2} h^{*}}{\partial x_{1}^{2}} u_{, 1}=q b
$$


Finally, by using (7), we can rewrite Eq. (5) as

$$
\omega_{p}\left(\frac{\partial h^{*}}{\partial x_{1}} u_{, 1}+h^{*} u_{, 11}\right) \mp c \gamma_{p} h_{0} h^{*}\left(2 \frac{\partial h^{*}}{\partial x_{1}} w_{, 11}+h^{*} w_{, 111}\right)=0 .
$$

It seems that Eqs. (14) and (15) cannot be decoupled and that both the deflection and axial displacement must be calculated from this system of the governing equations. Such a decoupling, however, can be accomplished if the depth of the beam is invariable (the derivatives of the depth disappear) even if Young's modulus is graded along $x_{3}$. Then, the solution of the bending problem in such a FGM beam is formally the same as in the homogeneous beam, but the bending stiffness is influenced by the coupling effect. Of course, the system is decoupled if the material of the beam is homogeneous, when the interaction constant $\gamma_{p}$ vanishes, even if the depth of the beam is variable.

According to (3) and (11), we obtain the expression for the shear stress

$$
T_{31}\left(x_{1}\right)=M_{2,1}\left(x_{1}\right)=-D_{0} D_{V}^{*}\left[\frac{\partial D_{H}^{*}}{\partial x_{1}}\left(x_{1}\right) w_{, 11}\left(x_{1}\right)+D_{H}^{*}\left(x_{1}\right) w_{, 111}\left(x_{1}\right)\right] \pm c \frac{\gamma_{p} h_{0}}{\omega_{p}} \frac{\partial h^{*}}{\partial x_{1}}\left(x_{1}\right) T_{11}\left(x_{1}\right) .
$$

Obviously, it is appropriate to solve the boundary value problems using a dimensionless formulation. By using the superscript star $(*)$ for the dimensionless quantities, we introduce the following definitions

$$
x_{1}=L x_{1}^{*}, \quad x_{3}=h_{0} x_{3}^{*}, \quad u=h_{0} u^{*}, \quad w=h_{0} w^{*} .
$$

Then, $w_{11}=\left(h_{0} / L\right) w_{1}^{*}, w_{11}=\left(h_{0} / L^{2}\right) w_{, 11}^{*}, \partial D_{H}^{*} / \partial x_{1}=D_{H, 1}^{*} / L, \partial^{2} D_{H}^{*} / \partial x_{1}^{2}=D_{H, 11}^{*} / L^{2}$ and the governing equations for the dimensionless field variables become

$$
\begin{gathered}
D_{V}^{*}\left(D_{H, 11}^{*} w_{, 11}^{*}+2 D_{H, 1}^{*} w_{, 111}^{*}+D_{H}^{*} w_{, 1111}^{*}\right)+12 \frac{\left(c \gamma_{p} h^{*}\right)^{2}}{\omega_{p}} h_{, 11}^{*} w_{, 11}^{*} \mp \frac{12 L}{h_{0}} c \gamma_{p} h^{*} h_{, 11}^{*} u_{, 1}^{*}=\frac{L^{4} b}{D_{0} h_{0}} q, \\
\omega_{p}\left(h_{, 1}^{*} u_{, 1}^{*}+h^{*} u_{, 11}^{*}\right) \mp c \gamma_{p} \frac{h_{0}}{L} h^{*}\left(2 h_{, 1}^{*} w_{, 11}^{*}+h^{*} w_{, 111}^{*}\right)=0
\end{gathered}
$$

The relevant boundary quantities for bending are $w$ (deflection), $w_{1}$ (normal slope of deflection), $M_{2}$ (bending moment) and $T_{31}$ (shear force), while for the in-plane deformation, these quantities are $u$ (axial displacement) and $T_{11}$ (axial traction), respectively. The dimensionless representations of these quantities are given by

$$
\begin{aligned}
& w_{, 1}^{*}\left(x_{1}^{*}\right)=\frac{d w^{*}\left(x_{1}^{*}\right)}{d x_{1}^{*}}, \quad w_{, 1}\left(x_{1}\right)=\frac{d w\left(x_{1}\right)}{d x_{1}}=\frac{h_{0}}{L} \frac{d w^{*}\left(x_{1}^{*}\right)}{d x_{1}^{*}}=\frac{h_{0}}{L} w_{, 1}^{*}\left(x_{1}^{*}\right) \\
& T_{11}^{*}\left(x_{1}^{*}\right)=\omega_{p} h^{*}\left(x_{1}^{*}\right) u_{, 1}^{*}\left(x_{1}^{*}\right) \mp c \frac{h_{0}}{L} \gamma_{p} h^{* 2}\left(x_{1}^{*}\right) w_{, 11}^{*}\left(x_{1}^{*}\right), \quad T_{11}\left(x_{1}\right)=12 \frac{D_{0}}{L h_{0}} T_{11}^{*}\left(x_{1}^{*}\right), \\
& M_{2}^{*}\left(x_{1}^{*}\right)=-D_{V}^{*} D_{H}^{*}\left(x_{1}^{*}\right) w_{, 11}^{*}\left(x_{1}^{*}\right) \pm c \frac{12 L}{h_{0}} \frac{\gamma_{p}}{\omega_{p}} h^{*}\left(x_{1}^{*}\right) T_{11}^{*}\left(x_{1}^{*}\right), \quad M_{2}\left(x_{1}\right)=\frac{D_{0} h_{0}}{L^{2}} M_{2}^{*}\left(x_{1}^{*}\right), \\
& T_{31}^{*}\left(x_{1}^{*}\right)=M_{2,1}^{*}\left(x_{1}^{*}\right)=-D_{V}^{*}\left[D_{H, 1}^{*}\left(x_{1}^{*}\right) w_{, 11}^{*}\left(x_{1}^{*}\right)+D_{H}^{*}\left(x_{1}^{*}\right) w_{, 111}^{*}\left(x_{1}^{*}\right)\right] \pm c \frac{12 L}{h_{0}} \frac{\gamma_{p}}{\omega_{p}} h_{1}^{*}\left(x_{1}^{*}\right) T_{11}^{*}\left(x_{1}^{*}\right), \quad T_{31}\left(x_{1}\right)=\frac{D_{0} h_{0}}{L^{3}} T_{31}^{*}\left(x_{1}^{*}\right) .
\end{aligned}
$$

Since the theory is linear, one can obtain the solution for the actual geometrical dimensions and loading by applying simple rescaling to the dimensionless solution.

\subsection{Decomposition of the governing equations}

It is well known that the higher order derivatives of field variables in governing equations give rise to difficulties in the solution of boundary value problems, because of the worse accuracy of the numerical evaluation of such derivatives. The order of the differential operator can be decreased mathematically by decomposing this operator into two lower order differential operators by introducing new field variables (Sladek et al., 2013). 
By introducing a new field variable $m$ by

$$
m\left(x_{1}\right):=-D w_{, 11}\left(x_{1}\right)=-\frac{D_{0} h_{0}}{L^{2}} D_{V}^{*} D_{H}^{*}\left(x_{1}^{*}\right) w_{, 11}^{*}\left(x_{1}^{*}\right)=\frac{D_{0} h_{0}}{L^{2}} m^{*}\left(x_{1}^{*}\right) \text { or } m^{*}\left(x_{1}^{*}\right):=-D_{V}^{*} D_{H}^{*}\left(x_{1}^{*}\right) w_{, 11}^{*}\left(x_{1}^{*}\right),
$$

the system of two differential equations (17) and (18) is split into a system of three ordinary differential equations (ODE):

$$
\begin{gathered}
D_{V}^{*} D_{H}^{*} w_{, 11}^{*}+m^{*}=0 \\
m_{, 11}^{*}+\frac{12}{D_{V}^{*} D_{H}^{*}} \frac{\left(c \gamma_{p} h^{*}\right)^{2}}{\omega_{p}} h_{, 11}^{*} m^{*} \pm \frac{12 L}{h_{0}} c \gamma_{p} h^{*} h_{, 11}^{*} u_{, 1}^{*}=-\lambda^{*} \\
\omega_{p}\left(h_{, 1}^{*} u_{, 1}^{*}+h^{*} u_{, 11}^{*}\right) \pm c \frac{\gamma_{p} h_{0}}{D_{V}^{*} L h^{* 2}}\left(h^{*} m_{, 1}^{*}-h_{, 1}^{*} m^{*}\right)=0
\end{gathered}
$$

where $\lambda^{*}\left(x^{*}\right)=\frac{L^{4} b}{D_{0} h_{0}} q(x)$ is the density of the dimensionless loading.

The relevant boundary quantities are expressed in the terms of the new field variables as

$$
\begin{gathered}
T_{11}^{*}\left(x_{1}^{*}\right)=\omega_{p} h^{*}\left(x_{1}^{*}\right) u_{, 1}^{*}\left(x_{1}^{*}\right) \pm c \frac{h_{0} \gamma_{p}}{D_{V}^{*} L} \frac{1}{h^{*}\left(x_{1}^{*}\right)} m^{*}\left(x_{1}^{*}\right), \\
M_{2}^{*}\left(x_{1}^{*}\right)=m^{*}\left(x_{1}^{*}\right) \pm 12 c \frac{L}{h_{0}} \frac{\gamma_{p}}{\omega_{p}} h^{*}\left(x_{1}^{*}\right) T_{11}^{*}\left(x_{1}^{*}\right), \\
T_{31}^{*}\left(x_{1}^{*}\right)=m_{, 1}^{*}\left(x_{1}^{*}\right) \pm 12 c \frac{L}{h_{0}} \frac{\gamma_{p}}{\omega_{p}} h_{1}^{*}\left(x_{1}^{*}\right) T_{11}^{*}\left(x_{1}^{*}\right) .
\end{gathered}
$$

Thus, one can solve the second order ODEs (25) and (27) with the prescribed boundary conditions for the coupled bending and axial deflection problem. If the decoupling is applicable $\left(\gamma_{p}=0\right.$ or $h=$ const), the axial deflection problem (with governing equation (27)) is solved after having solved the bending problem described by the system of ODEs (25)-(26).

\section{MOVING LEAST SQUARES (MLS) APPROXIMATION SCHEME}

In the MLS approximation, the polynomial basis $\left\{p_{\mu}(\mathbf{x})\right\}_{\mu=1}^{m}$ is employed, and the expansion coefficients are found from a minimization of the weighted squares of the residue at a finite number of nodal points (Lancaster and Salkauskas, 1981). Finally, the scalar field $u(\mathbf{x})$ can be approximated as

$$
u(\mathbf{x}) \approx \sum_{a=1}^{N} \hat{u}^{a} \phi^{a}(\mathbf{x})
$$

where $N$ is the total number of nodes; $\hat{u}^{a}$ is a nodal unknown different from the nodal value $u\left(\mathbf{x}^{a}\right)$; and $\phi^{a}(\mathbf{x})$ is the shape function associated with the nodal point $\mathbf{x}^{a}$. Instead of the standard MLS approximation, one can utilize the Central Approximation Node (CAN) concept of the MLS approximation (Sladek, Sladek and Zhang, 2008). Let $\mathbf{x}^{q}$ be the CAN for the approximation at a point $\mathbf{x}$. Then, the amount of nodes involved in the approximation at $\mathbf{x}$ is reduced a priori from $N$ to $N^{q}$ where $N^{q}$ is the number of nodes supporting the approximation at the CAN $\mathbf{x}^{q}$, i.e., the amount of nodes in the $\operatorname{set} M{ }^{q}=\left\{\forall \mathbf{x}^{a} ; w^{a}\left(\mathbf{x}^{q}\right)>0\right\}_{a=1}^{N}$, where $w^{a}(\mathbf{x})$ is the weight function associated with the node $\mathbf{x}^{a}$ and taken at the field point $\mathbf{x}$. In this paper, we employ the Gaussian weights (Sladek, Sladek and Zhang, 2008). The MLS-CAN approximation is given as

$$
u(\mathbf{x}) \approx \sum_{a=1}^{N^{q}} \hat{u}^{\bar{a}} \phi^{(q, a)}(\mathbf{x}), \bar{a}=n(q, a),
$$

where $\bar{a}$ is the global number of the $a$-th node from the $N^{q}$ nodal points $\mathbf{x}^{\bar{a}} \in M^{q}$. The CAN node can be selected as the nearest node to the field point $\mathbf{x}$. 
The derivatives of the field variable $u(\mathbf{x})$ can be approximated in a standard way by differentiating the approximation (32), i.e.,

$$
u_{, i}(\mathbf{x}) \approx \sum_{a=1}^{N^{q}} \hat{u}^{\bar{a}} \phi_{, i}^{(q, a)}(\mathbf{x}), \quad u_{, i j}(\mathbf{x}) \approx \sum_{a=1}^{N^{q}} \hat{u}^{\bar{a}} \phi_{, i j}^{(q, a)}(\mathbf{x}), \quad u_{, i j k}(\mathbf{x}) \approx \sum_{a=1}^{N^{q}} \hat{u}^{\bar{a}} \phi_{, i j k}^{(q, a)}(\mathbf{x}) .
$$

Besides the standard differentiation approach (denoted as "D0") we shall also use a modified differentiation (denoted as the D1 approach) in the calculation of the derivatives of the field variables. In this approach, the derivatives are approximated by using the shape functions $\phi^{\bar{a}}(\mathbf{x})$ and certain nodal values, which will be expressed in terms of the nodal values $\hat{u}^{h}$ and the first order derivatives of the shape functions. Thus,

$$
u_{, i}(\mathbf{x}) \approx \sum_{a=1}^{N^{q}} \hat{u}_{i}^{\bar{a}} \phi^{(q, a)}(\mathbf{x}), \quad u_{, i j}(\mathbf{x}) \approx \sum_{a=1}^{N^{q}} \hat{u}_{i j}^{\bar{a}} \phi^{(q, a)}(\mathbf{x}), \quad u_{, j j k}(\mathbf{x}) \approx \sum_{a=1}^{N^{q}} \hat{u}_{i j k}^{\bar{a}} \phi^{(q, a)}(\mathbf{x}) .
$$

From (33 $)$, we have

$$
u_{, i}\left(\mathbf{x}^{c}\right) \approx \sum_{h=1}^{N^{c}} \hat{u}^{\bar{h}} \phi_{, i}^{(c, h)}\left(\mathbf{x}^{c}\right)=\sum_{h=1}^{N^{c}} f_{i}^{c h} \hat{u}^{\bar{h}}, \text { with } \quad \bar{h}=n(c, h), f_{i}^{c h}=\phi_{, i}^{(c, h)}\left(\mathbf{x}^{c}\right),
$$

while from $\left(34_{1}\right)$, we have

$$
u_{, i}\left(\mathbf{x}^{c}\right) \approx \sum_{a=1}^{N^{c}} \hat{u}_{i}^{\bar{a}} \phi^{(c, a)}\left(\mathbf{x}^{c}\right)=\sum_{h=1}^{N^{c}} e^{c a} \hat{u}_{i}^{\bar{a}}, \text { with } \bar{a}=n(c, a), e^{c a}=\phi^{(c, a)}\left(\mathbf{x}^{c}\right) .
$$

Extending the definitions of matrices $f_{i}^{c h}$ and $e^{c a}$ to all the nodes as

$$
E^{c d}:=\left\{\begin{array}{l}
e^{c a}, d=\bar{a} \\
0, d \neq \bar{a}
\end{array}, \quad F_{i}^{c g}:=\left\{\begin{array}{c}
f_{i}^{c a}, g=\bar{a} \\
0, g \neq \bar{a}
\end{array} \text {, with } \bar{a}=n(c, a), a \in\left\{1,2, \ldots, N^{c}\right\},,\right.\right.
$$

we can rewrite Eqs. (35) and (36) as

$$
u_{, i}\left(\mathbf{x}^{c}\right)=\sum_{d=1}^{N} E^{c d} \hat{u}_{i}^{d}=\sum_{g=1}^{N} F_{i}^{c g} \hat{u}^{g}
$$

hence,

$$
\hat{u}_{i}^{d}=\sum_{g=1}^{N} \sum_{c=1}^{N}\left(E^{-1}\right)^{d c} F_{i}^{c g} \hat{u}^{g}=\sum_{g=1}^{N} G_{i}^{d g} \hat{u}^{g}, \quad G_{i}^{d g}:=\sum_{c=1}^{N}\left(E^{-1}\right)^{d c} F_{i}^{c g} .
$$

Differentiating $\left(34_{1}\right)$, we obtain

From $\left(34_{2}\right)$, we have

$$
u_{, i j}(\mathbf{x}) \approx \sum_{a=1}^{N^{q}} \hat{u}_{i}^{\bar{a}} \phi_{, j}^{(q, a)}(\mathbf{x}) \Rightarrow u_{, i j}\left(\mathbf{x}^{c}\right) \approx \sum_{a=1}^{N^{c}} \hat{u}_{i}^{\bar{a}} \phi_{, j}^{(c, a)}\left(\mathbf{x}^{c}\right)=\sum_{g=1}^{N} F_{j}^{c g} \hat{u}_{i}^{g} .
$$

$$
u_{, i j}\left(\mathbf{x}^{c}\right) \approx \sum_{a=1}^{N^{c}} \hat{u}_{i j}^{\bar{a}} \phi^{(c, a)}\left(\mathbf{x}^{c}\right)=\sum_{d=1}^{N} E^{c d} \hat{u}_{i j}^{d}
$$

and from a comparison of (40) with (41), we receive

$$
\hat{u}_{i j}^{d}=\sum_{g=1}^{N} \sum_{c=1}^{N}\left(E^{-1}\right)^{d c} F_{j}^{c g} \hat{u}_{i}^{g}=\sum_{g=1}^{N} G_{j}^{d g} \hat{u}_{i}^{g}=\sum_{g, h=1}^{N} G_{j}^{d g} G_{i}^{g h} \hat{u}^{h},
$$

where in the last equality, we have utilized the expression for $\hat{u}_{i}^{g}$ by (39).

Similarly,

$$
\hat{u}_{i j k}^{d}=\sum_{g, b, h=1}^{N} G_{k}^{d g} G_{j}^{g b} G_{i}^{b h} \hat{u}^{h}
$$


Thus, having the expressions of $\hat{u}_{i}^{d}, \hat{u}_{i j}^{d}, \hat{u}_{i j k}^{d}$ from Eqs. (39), (42) and (43), one can utilize (42) for a modified representation of the derivatives of the field variables in terms of the shape function $\phi^{a}(\mathbf{x})$ and all the nodal values $\hat{u}^{h}$. In the D1 approach, only the first order derivatives at the nodal points are employed in contrast to the standard D0 approach. Since the inversion of the matrix $E^{c d}$ is needed in the evaluation of the matrix $G_{i}^{d g}$ defined by (39), the lower computational efficiency of the D1 approach as compared with the D0 approach is the price which should be paid for the better accuracy of the D1 approach.

Furthermore, it is worth considering the modification of the shape functions and their derivatives. Making use of the definitions

$$
s(\mathbf{x}):=\sum_{a=1}^{N^{q}} \phi^{(q, a)}(\mathbf{x}), \quad s_{i}(\mathbf{x}):=\sum_{a=1}^{N^{q}} \phi_{, i}^{(q, a)}(\mathbf{x}), \quad s_{i j}(\mathbf{x}):=\sum_{a=1}^{N^{q}} \phi_{, i j}^{(q, a)}(\mathbf{x}), \quad s_{i j k}(\mathbf{x}):=\sum_{a=1}^{N^{q}} \phi_{, j j k}^{(q, a)}(\mathbf{x}),
$$

and adopting the modifications

$$
\begin{aligned}
& \phi^{(q, a)}(\mathbf{x}) \rightarrow \tilde{\phi}^{(q, a)}(\mathbf{x}):=\phi^{(q, a)}(\mathbf{x}) / s(\mathbf{x}), \phi_{, i}^{(q, a)}(\mathbf{x}) \rightarrow \tilde{\phi}_{, i}^{(q, a)}(\mathbf{x}):=\phi_{, i}^{(q, a)}(\mathbf{x})-s_{i}(\mathbf{x}) \tilde{\phi}^{(q, a)}(\mathbf{x}), \\
& \phi_{, i j}^{(q, a)}(\mathbf{x}) \rightarrow \tilde{\phi}_{, i j}^{(q, a)}(\mathbf{x}):=\phi_{, i j}^{(q, a)}(\mathbf{x})-s_{i j}(\mathbf{x}) \tilde{\phi}^{(q, a)}(\mathbf{x}), \\
& \phi_{, i j k}^{(q, a)}(\mathbf{x}) \rightarrow \tilde{\phi}_{, i j k}^{(q, a)}(\mathbf{x}):=\phi_{, i j k}^{(q, a)}(\mathbf{x})-s_{i j k}(\mathbf{x}) \tilde{\phi}^{(q, a)}(\mathbf{x}),
\end{aligned}
$$

one can guarantee the satisfaction of the following equations

$$
\sum_{a=1}^{N^{q}} \phi^{(q, a)}(\mathbf{x})=1, \quad \sum_{a=1}^{N^{q}} \phi_{, i}^{(q, a)}(\mathbf{x})=0, \quad \sum_{a=1}^{N^{q}} \phi_{, i j}^{(q, a)}(\mathbf{x})=0, \quad \sum_{a=1}^{N^{q}} \phi_{, j j k}^{(q, a)}(\mathbf{x})=0
$$

with the wave notation being omitted. The formulation using the standard shape functions will be denoted as the S0 approach in contrast to the S1 approach utilizing the modified shape functions.

\section{STRONG FORMULATION OF THE GOVERNING EQUATIONS}

It is well known that the utilization of a meshless approximation for field variables is only necessary but not sufficient for getting a truly meshless formulation for the solution of boundary value problems. If the governing equations are satisfied in the weak (or integral) sense on the whole domain analyzed, a background mesh is still needed to accomplish the integrations. On the other hand, considerations of weak forms on local subdomains yield truly meshless formulations (Atluri, 2004). Since in strong formulations, the governing equations are satisfied at the collocation points, the utilization of meshless approximations guarantees a truly meshless formation. Nevertheless, there may be significant differences among the various meshless strong formulations. If a global meshless approximation is employed, the resulting system matrix is fully populated in contrast to the case of local meshless approximations leading to sparse system matrices. Usually, the collocation points are identical with the nodal points used for the approximation of field variables. Although the choice of the test functions in the weak formulations in view of the Dirac $\delta$-functions enables us to classify the strong formulation as a special case of the Petrov-Galerkin method, this is just a formal act because the strong formulation is not actually the formulation in an integral sense.

In enforcing the satisfaction of the governing equations (25)-(27) at the interior nodes $\mathbf{x}^{c}$, one obtains the system of ODEs:

$$
\begin{gathered}
D_{V}^{*} D_{H}^{*} w_{, 11}^{*}\left(x^{* c}\right)+m^{*}\left(x^{* c}\right)=0, \\
m_{, 11}^{*}\left(x^{* c}\right)+\frac{12}{D_{V}^{*} D_{H}^{*}} \frac{\left(c \gamma_{p} h^{*}\left(x^{* c}\right)\right)^{2}}{\omega_{p}} h_{, 11}^{*}\left(x^{* c}\right) m^{*}\left(x^{* c}\right) \pm \frac{12 L}{h_{0}} c \gamma_{p} h^{*}\left(x^{* c}\right) h_{, 11}^{*}\left(x^{* c}\right) u_{, 1}^{*}\left(x^{* c}\right)=-\lambda^{*}\left(x^{* c}\right), \\
\omega_{p}\left[h_{, 1}^{*}\left(x^{* c}\right) u_{, 1}^{*}\left(x^{* c}\right)+h^{*}\left(x^{* c}\right) u_{, 11}^{*}\left(x^{*} c\right)\right] \pm \frac{c \gamma_{p} h_{0}}{D_{V}^{*} L\left(h^{*}\left(x^{*} c\right)\right)^{2}}\left[h^{*}\left(x^{* c}\right) m_{, 1}^{*}\left(x^{* c}\right)-h_{, 1}^{*}\left(x^{* c}\right) m^{*}\left(x^{* c}\right)\right]=0 .
\end{gathered}
$$

Hence in view of the MLS-CAN approximation for the field variables $w^{*}, m^{*}, u^{*}$, the system of discretized governing equations is given as

$$
D \sum_{a=1}^{N^{c}} \phi_{, 11}^{(c, a)}\left(x^{* c}\right) \hat{w}^{* \bar{a}}+\sum_{a=1}^{N^{c}} \phi^{(c, a)}\left(x^{* c}\right) \hat{m}^{* \bar{a}}=0
$$




$$
\begin{gathered}
\sum_{a=1}^{N^{c}}\left[\frac{12}{D_{V}^{*} D_{H}^{*}} \frac{\left(c \gamma_{p} h^{* c}\right)^{2}}{\omega_{p}} h_{, 11}^{* c} \phi^{(c, a)}\left(x^{* c}\right)+\phi_{, 11}^{(c, a)}\left(x^{* c}\right)\right] \hat{m}^{* \bar{a}} \pm \frac{12 L}{h_{0}} c \gamma_{p} h^{* c} h_{, 11}^{* c} \sum_{a=1}^{N^{c}} \phi_{, 1}^{(c, a)}\left(x^{* c}\right) \hat{u}^{* \bar{a}}=-\lambda^{*}\left(x^{* c}\right), \\
\omega_{p} \sum_{a=1}^{N^{c}}\left[h_{, 1}^{* c} \phi_{, 1}^{(c, a)}\left(x^{* c}\right)+h^{* c} \phi_{, 11}^{(c, a)}\left(x^{* c}\right)\right] \hat{u}^{* \bar{a}} \pm \frac{c \gamma_{p} h_{0}}{D_{V}^{*} L\left(h^{* c}\right)^{2}} \sum_{a=1}^{N^{c}}\left[h^{* c} \phi_{, 1}^{(c, a)}\left(x^{* c}\right)-h_{, 1}^{* c} \phi^{(c, a)}\left(x^{* c}\right)\right] \hat{m}^{* \bar{a}}=0
\end{gathered}
$$

for $x^{c} \in \Omega$. For the sake of brevity, we have used short notations $h^{* c}=h^{*}\left(x^{* c}\right), h_{1}^{* c}=h_{1}^{*}\left(x^{* c}\right)$, etc.

With regard to the discretized boundary conditions, we shall distinguish three events at $x_{1}^{* b} \in\left\{x_{1}^{*}=0, x_{1}^{*}=1\right\}$ :

(i) clamped end $x_{1}^{b}: w^{*}\left(x_{1}^{* b}\right)=0, w_{, 1}^{*}\left(x_{1}^{* b}\right)=0, u^{*}\left(x_{1}^{* b}\right)=0$ hence,

$$
\sum_{a=1}^{N^{b}} \phi^{(b, a)}\left(x_{1}^{* b}\right) \hat{w}^{* \bar{a}}=0, \quad \sum_{a=1}^{N^{b}} \phi_{, 1}^{(b, a)}\left(x_{1}^{* b}\right) \hat{w}^{* \bar{a}}=0, \quad \sum_{a=1}^{N^{b}} \phi^{(b, a)}\left(x_{1}^{* b}\right) \hat{u}^{* \bar{a}}=0,
$$

(ii) simply supported end $x_{1}^{b}: w^{*}\left(x_{1}^{* b}\right)=0, M_{2}^{*}\left(x_{1}^{* b}\right)=m^{*}\left(x_{1}^{* b}\right)=0, T_{11}^{*}\left(x_{1}^{* b}\right)=0$ hence according to (28)-(29),

$$
\begin{aligned}
& \sum_{a=1}^{N^{b}} \phi^{(b, a)}\left(x_{1}^{* b}\right) \hat{w}^{* \bar{a}}=0, \quad \sum_{a=1}^{N^{b}} \phi^{(b, a)}\left(x_{1}^{* b}\right) \hat{m}^{* \bar{a}}=0, \\
& \sum_{a=1}^{N^{b}}\left\{\omega_{p} h^{*}\left(x_{1}^{* b}\right) \phi_{, 1}^{(b, a)}\left(x_{1}^{* b}\right) \hat{u}^{* \bar{a}} \pm c \frac{h_{0} \gamma_{p}}{D_{V}^{*} L} \frac{1}{h^{*}\left(x_{1}^{* b}\right)} \phi^{(b, a)}\left(x_{1}^{* b}\right) \hat{m}^{* \bar{a}}\right\}=0,
\end{aligned}
$$

(iii) free end $x_{1}^{b}: \quad M_{2}^{*}\left(x_{1}^{* b}\right)=m^{*}\left(x_{1}^{* b}\right)=0, T_{31}^{*}\left(x_{1}^{* b}\right)=m_{, 1}^{*}\left(x_{1}^{* b}\right)=0, \quad T_{11}^{*}\left(x_{1}^{* b}\right)=0$ hence according to (28)-(30),

$$
\begin{aligned}
& \sum_{a=1}^{N^{b}} \phi^{(b, a)}\left(x_{1}^{* b}\right) \hat{m}^{* \bar{a}}=0, \quad \sum_{a=1}^{N^{b}} \phi_{, 1}^{(b, a)}\left(x_{1}^{* b}\right) \hat{m}^{* \bar{a}}=0, \\
& \sum_{a=1}^{N^{b}}\left\{\omega_{p} h^{*}\left(x_{1}^{* b}\right) \phi_{, 1}^{(b, a)}\left(x_{1}^{* b}\right) \hat{u}^{* \bar{a}} \pm c \frac{h_{0} \gamma_{p}}{D_{V}^{*} L} \frac{1}{h^{*}\left(x_{1}^{* b}\right)} \phi^{(b, a)}\left(x_{1}^{* b}\right) \hat{m}^{* \bar{a}}\right\}=0 .
\end{aligned}
$$

Recall that the axial rigid body motion must be eliminated, if both ends of the beam are free of the axial force $T_{11}^{*}\left(x_{1}^{* b}\right)=0$.

\section{NUMERICAL EXAMPLES}

In this section, some numerical results of the deflections and axial displacements of the FGM beams with specified material properties, geometry, applied load and boundary conditions are presented. A rectangular beam with a transversal gradation of Young's modulus is analyzed. The FGM beam considered has a length of $L=1 \mathrm{~m}$, a width of $b_{0}=0.05 \mathrm{~m}$, and a depth of $h_{0}=0.1 \mathrm{~m}$. Furthermore, we assume the uniform static loading $\lambda_{0}^{*}=1$ and the dimensionless Young's modulus and variable depth are prescribed by Eqs. (1) and (9), respectively. Poisson's ratio is $v=0.3$, while various levels of the gradation $(\zeta, \kappa)$ and various values of the power-law gradation exponents $(p, s)$ are considered. The exact solutions of the governing equations are derived in the Appendix for the FGM beams with invariable depths, and they shall be used for an investigation of the accuracy, the convergence of the accuracy as well as the computational efficiency of the meshless method presented. The accuracy of the numerical solutions of the boundary value problems will be characterized by an error norm defined as

$$
\text { error norm }=100 \frac{\left(\sum_{a=1}^{N}\left\{\left[w\left(x^{a}\right)-w^{e x}\left(x^{a}\right)\right]^{2}+\left[u\left(x^{a}\right)-u^{e x}\left(x^{a}\right)\right]^{2}\right\}\right)^{1 / 2}}{\left(\sum_{a=1}^{N}\left\{\left[w^{e x}\left(x^{a}\right)\right]^{2}+\left[u^{e x}\left(x^{a}\right)\right]^{2}\right\}\right)^{1 / 2}}(\%) \text {, }
$$

where $N$ is the total number of nodal points; $w\left(x^{a}\right)$, and $u\left(x^{a}\right)$ are the values of the field variables computed by the present method, while $w^{e x}\left(x^{a}\right)$ and $u^{e x}\left(x^{a}\right)$ are the exact values at the nodal points $x^{a}$.

In all the numerical computations, we have used a uniform distribution of the nodal points and the radius of the sub-domain, $\rho_{O}=0.1 \delta$ with $\delta$ being the distance between two neighbouring nodes. The other parameters in the MLS approximation have been taken as: radius of the interpolation domain $\rho^{a}=3.001 \delta$, shape function parameter $c^{a}=\delta$, and cubic polynomial basis $m=4$. The domain analyzed is $\Omega=\{\forall x ; x \in[0, L]\}$. 
Firstly, we pay attention to the study of the accuracy, the convergence of the accuracy and the computational efficiency. As an illustration we selected results corresponding to a beam with both ends being clamped $\left(w(0)=0, w_{1}(0)=0, u(0)=0\right.$; and $\left.w(L)=0, w_{1}(L)=0, u(L)=0\right)$ and the following values for the level $\zeta$ and exponents $p$ of the transversal power-law gradation of Young's modulus:

(i) $(\zeta=1, p=1)$,

(ii) $(\zeta=5, p=1)$,

(iii) $(\zeta=5, p=5)$.

The variation of Young's modulus is presented in Figure 1. The accuracy and convergence of the accuracy with respect to decreasing the $\delta$ - parameter (increasing the density of the nodes) are shown in Figure 2. It can be seen that the collocation formulation exhibits convergent and highly accurate numerical solutions. The influence of the S1 modification for an evaluation of the shape functions and their derivatives on the accuracy is negligible, while the improvement of the accuracy due to the modified differentiation (D1 approach) is significant compared with the standard differentiation. The convergence rate by the D1 approach is slightly better than that of the D0 approach, but both approaches yield very precise results.

It is interesting to compare the computational efficiency of the computational schemes discussed and employed. Note that the computational times are almost invariable with respect to the different boundary value problems, while in the case of large amounts of nodes, a substantial difference is observed (Figure 4) for the rates of increasing the CPU by increasing the density of the nodes when the D0 and the D1 approaches are used for the evaluation of the $2^{\text {nd }}$ order derivatives of the field variables. The utilization of the D1 approach brings about a significant enhancement of the rate of the CPU's time increase. The prolongation of the CPU time is due to a more complex procedure for evaluating the derivatives of the field variables than in the case of the D0 approach. Such an increase in the CPU time appears to be an unacceptable price for improving the accuracy of the numerical solutions by using the D1 approach in formulations for the decomposed problem of bending the beams with a transversal gradation of the Young's modulus.

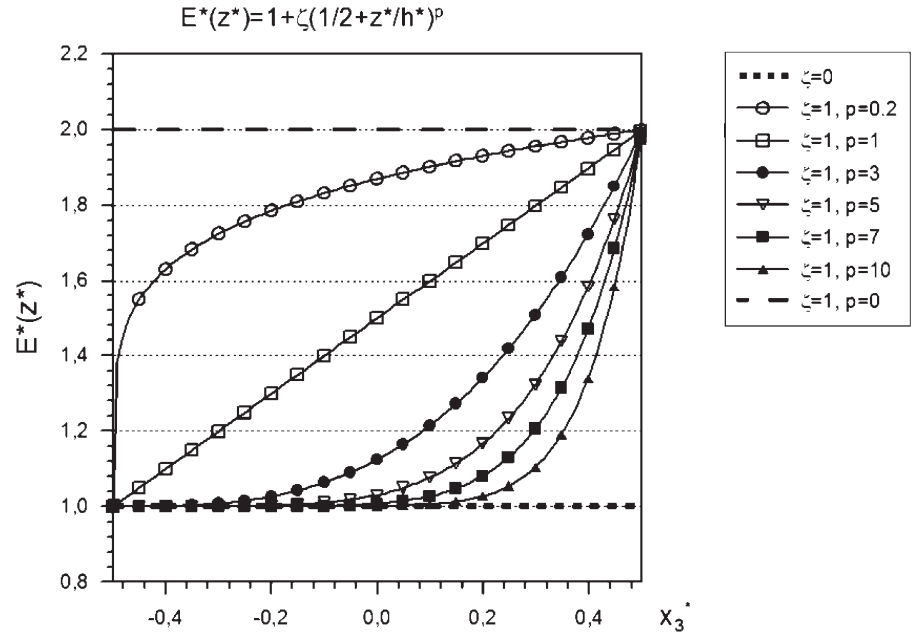

Fig. 1 Variation of dimensionless Young's modulus across the beam's depth with a power-law gradation.

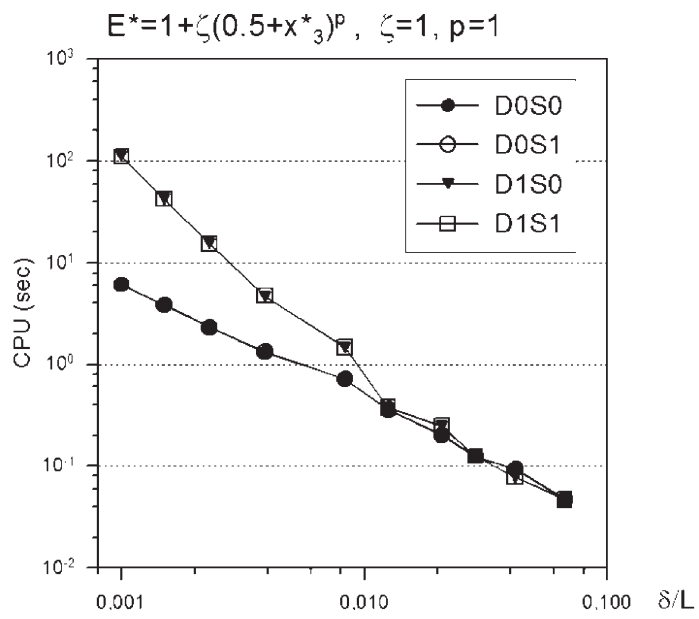

Fig. 3 Comparison of the computational times for four approximation techniques, depending on $\delta$-parameter.
$E^{*}=1+\zeta\left(0.5+x^{*}\right)^{p}, \zeta=1, p=1$

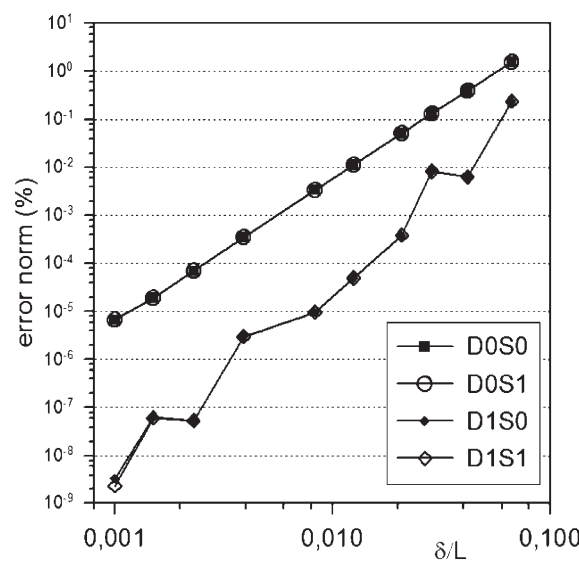

$E^{*}=1+\zeta\left(0.5+x^{*}\right)^{p}, \zeta=5, p=1$

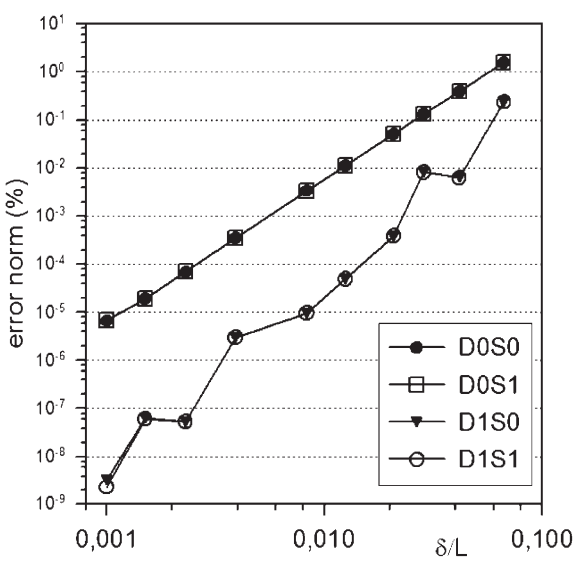

$E^{*}=1+\zeta\left(0.5+x^{*}\right)^{p}, \zeta=5, p=5$

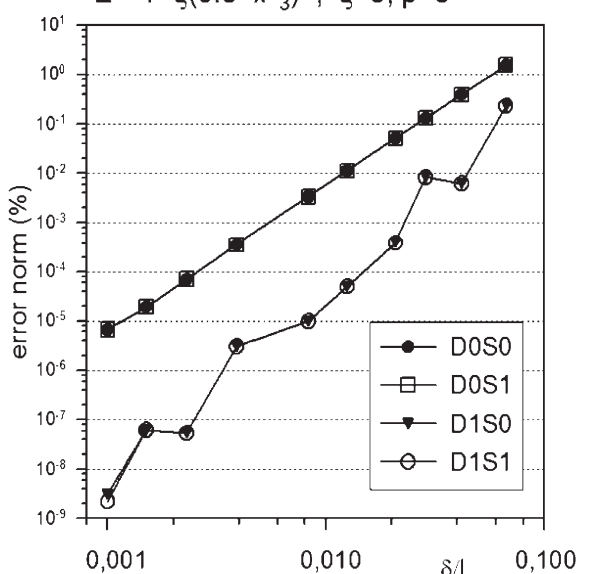

Fig. 2 Convergence study of a beam with a constant depth and transversally variable Young's modulus. 
In the following, we shall also allow for the longitudinal power-law gradation of the beam's depth (Figure 4) beside the transversal gradation of Young's modulus. The numerical results will be presented for three boundary value problems (BVP):

(i) both ends are clamped $\left(w(0)=0, w_{I}(0)=0, u(0)=0\right.$; and $\left.w(L)=0, w_{I}(L)=0, u(L)=0\right)$,

(ii) the left end is clamped $\left(w(0)=0, w_{, I}(0)=0, u(0)=0\right)$, and the right end is simply supported $(w(L)=0$, $\left.M_{2}(L)=0, T_{11}(L)=0\right)$,

(iii) the left end is clamped $\left(w(0)=0, w_{, I}(0)=0, u(0)=0\right)$, and the right end is free $\left(M_{2}(L)=0, T_{3 l}(L)=0\right.$,

$$
\left.T_{11}(L)=0\right) \text {. }
$$

Figure 5 illustrates the influence of the level of the gradation of Young's modulus $(\zeta=0$ or $\zeta \neq 0)$ and the beam's depth $(\kappa=0$ or $\kappa \neq 0)$ onto the deflections of the FGM beam.

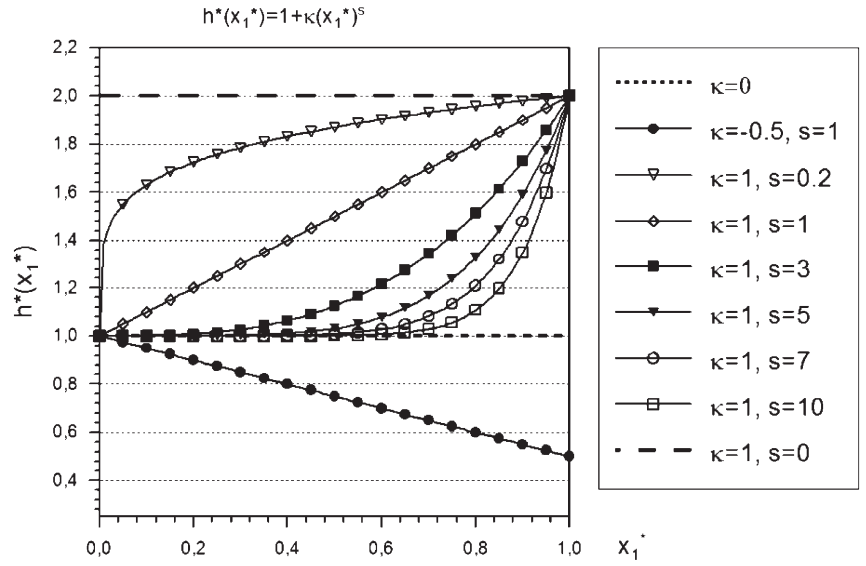

Fig. 4 Axial dependence of the dimensionless depth in a beam with a power-law graded depth.

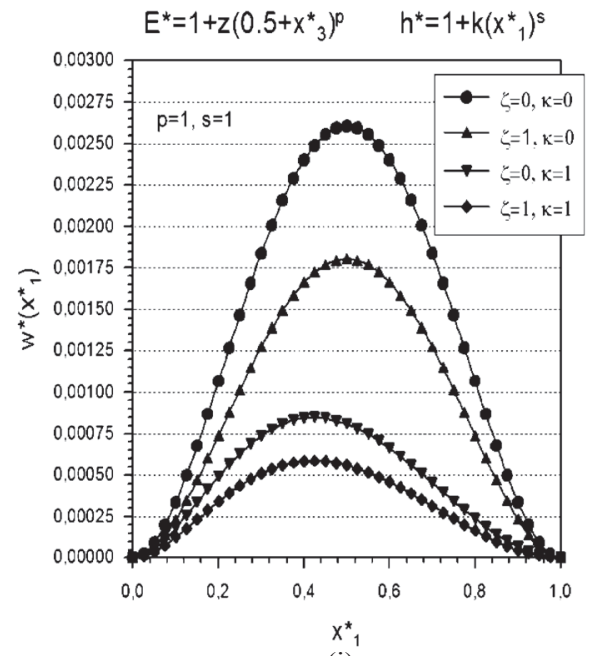

(i)

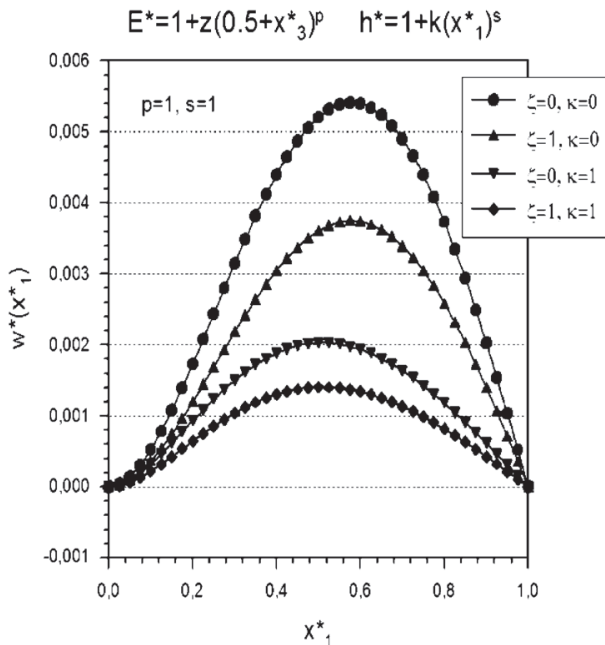

(ii)

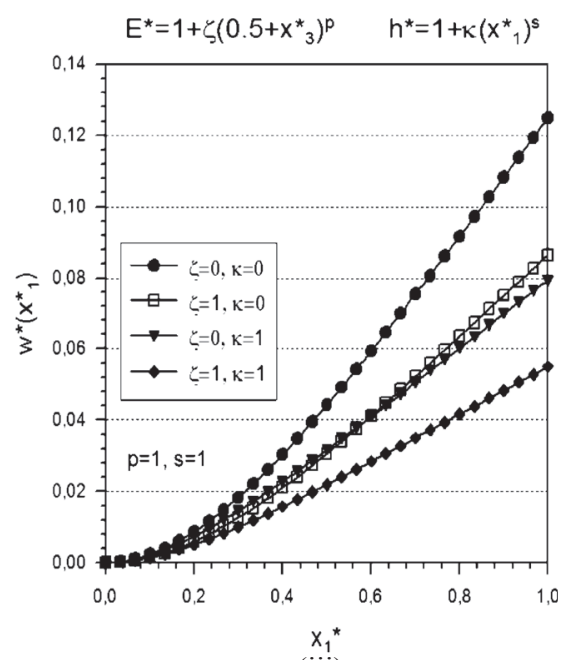

(iii)

Fig. 5 Axial distributions of the dimensionless deflections of a homogeneous $(\zeta=0)$ as well as an $F G M(\zeta \neq 0)$ beam of constant $(\kappa=0)$ and or variable $(\kappa \neq 0)$ depth by assuming the linear gradation in three b.v.p.

It is interesting to investigate the influence of the gradation parameters $(\zeta, \kappa$ and $p)$ on the response of the beams subjected to static loading. Figure 6 shows the numerical results for the deflections, axial displacement, bending moment, transversal shear force and normal stresses in a thinning beam $(\kappa<0)$ when intensifying Young's modulus $(\zeta>0)$ according to the transversal linear gradation. The influence of the exponent of the transversal power-law gradation of Young's modulus on the dimensionless deflections is presented in Figures 6-7. It can be seen that the influence of the parameters of gradation is significant on the magnitude as well as on the axial distribution of the physical quantities.

The role of coupling between the deflections and axial displacements on the reduction of deflections has been studied too. Figure 8 shows the dependencies of the reductions of the maximal deflections on the gradation parameters $\zeta$ (the level of the linear transversal gradation of Young's modulus), $p$ (the exponent of the transversal power-law gradation of Young's modulus), and $s$ (the exponent of the axial power-law gradation of the beam's depth). It can be seen that the volume content of the constituent with a higher Young's modulus is decreasing when increasing the exponent of the power-law gradation of Young's modulus; hence, the reduction of $W_{\max }$ (due to the transversal gradation of $E$ ) is increasing when increasing this exponent. 

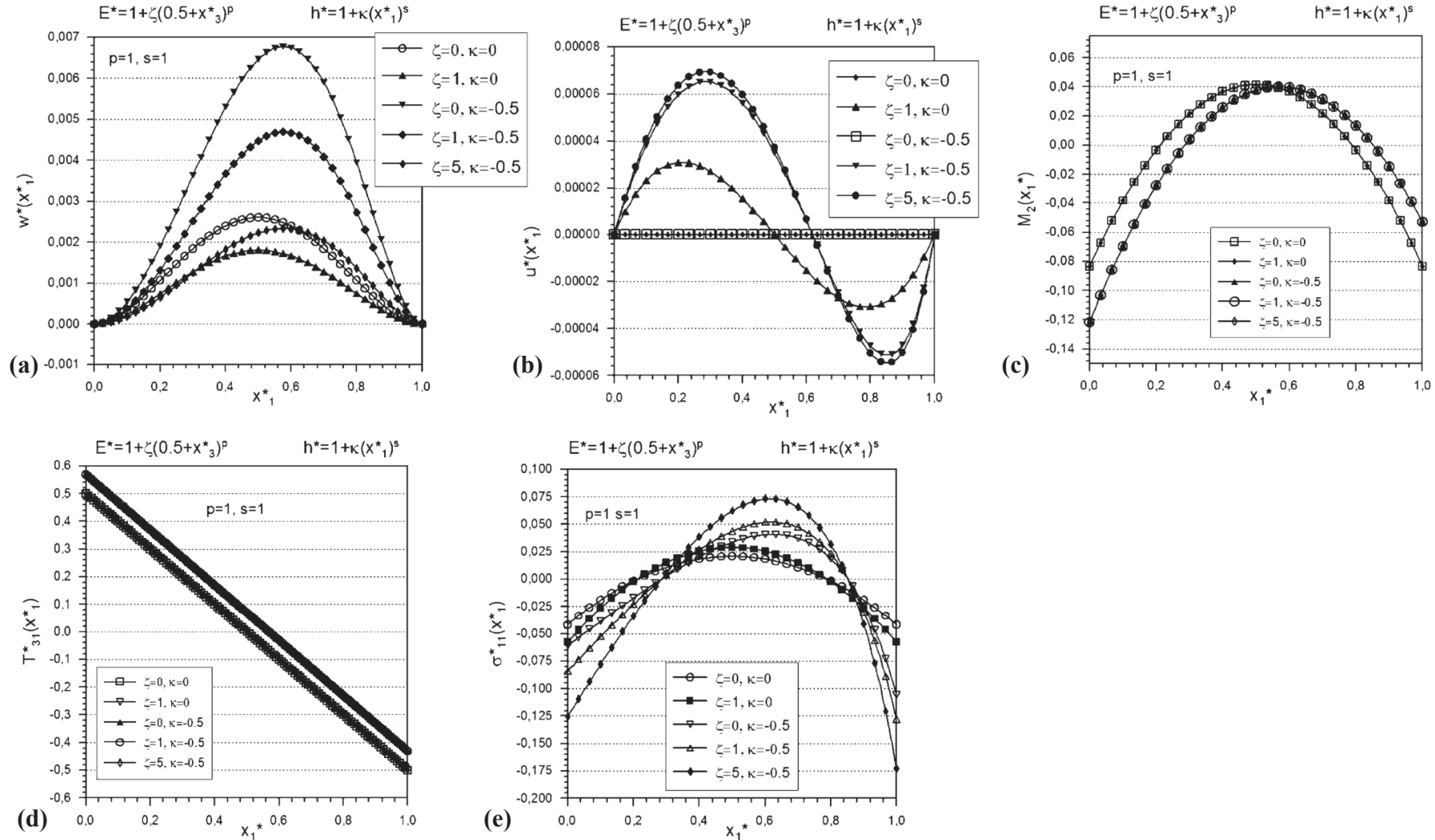

Fig. 6 Axial distributions of the dimensionless (a) deflections $w^{*}$, (b) axial displacements $u^{*}$, (c) bending moment $M_{2}^{*}$, (d) transversal shear force $T_{31}^{*}$, and (e) stresses $\sigma_{31}^{*}$ in beams with clamped ends.

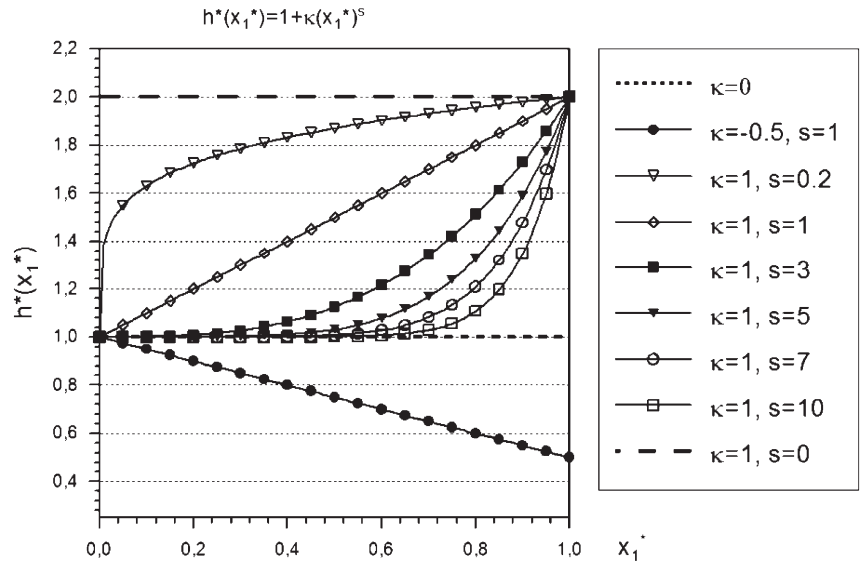

Fig. 7 Influence of the exponent of the transversal power-law gradation of Young's modulus.
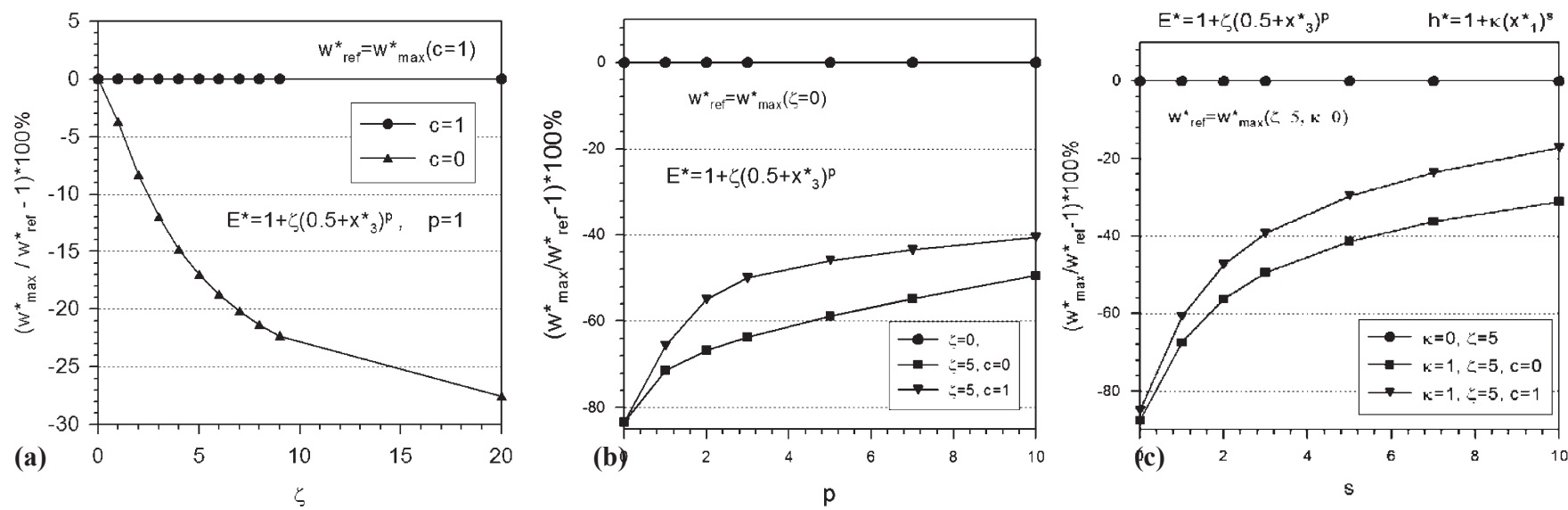

Fig. 8 Influence of $w-u$ coupling on the maximum deflection of the beam. 


\section{CONCLUSIONS}

The decomposition of a coupled FGM beam bending-problem governed by a system of two ODEs with a biharmonic operator into a problem governed by three coupled ODEs with lower order derivatives is developed and discussed. The strong (collocation) formulation combined with a meshless approximation technique is employed in the solution of the boundary value problems for bending the beams with a transversal gradation of Young's modulus and a variable depth. The MLS-CAN approximation scheme is adopted for the approximation of the physical quantities. The computational technique developed is verified in numerical tests involving the accuracy, convergence and computational efficiency of the study. The role of the coupling between the deflection and axial displacement is pointed out and illustrated in numerical simulations. The influence of the gradation parameters on the response of the FGM beams was investigated and discussed.

\section{Appendix}

In this Appendix, we derive the exact solutions for the beam with a transversal gradation of Young's modulus and an invariable depth $\left(h^{*}=1\right)$ Eliminating the field variable $m^{*}\left(x^{*}\right)$ from the governing equations (25)-(27), one obtains the decoupled set of governing equations

$$
D_{V}^{*} w_{, 1111}^{*}\left(x^{*}\right)=\lambda^{*}\left(x^{*}\right), \quad u_{, 11}^{*}\left(x^{*}\right)= \pm \frac{\gamma_{p} h_{0}}{\omega_{p} L} w_{, 111}^{*}\left(x^{*}\right)
$$

The general solutions of the governing equations (A.1) are:

$$
\begin{gathered}
w_{, 111}^{*}\left(x_{1}^{*}\right)=\frac{\lambda^{*}}{D_{V}^{*}} x_{1}^{*}+C_{1}, \\
w_{, 11}^{*}\left(x_{1}^{*}\right)=\frac{\lambda^{*}}{2 D_{V}^{*}}\left(x_{1}^{*}\right)^{2}+C_{1} x_{1}^{*}+C_{2}=-\frac{m^{*}\left(x_{1}^{*}\right)}{D_{V}^{*}}, \\
w_{, 1}^{*}\left(x_{1}^{*}\right)=\frac{\lambda^{*}}{6 D_{V}^{*}}\left(x_{1}^{*}\right)^{3}+\frac{C_{1}}{2}\left(x_{1}^{*}\right)^{2}+C_{2} x_{1}^{*}+C_{3}, \\
w^{*}\left(x_{1}^{*}\right)=\frac{\lambda^{*}}{24 D_{V}^{*}}\left(x_{1}^{*}\right)^{4}+\frac{C_{1}}{6}\left(x_{1}^{*}\right)^{3}+\frac{C_{1}}{2}\left(x_{1}^{*}\right)^{2}+C_{3} x_{1}^{*}+C_{4}, \\
u_{, 1}^{*}\left(x_{1}^{*}\right)= \pm \frac{\gamma_{p} h_{0}}{\omega_{p} L}\left(\frac{\lambda^{*}}{2 D_{V}^{*}}\left(x_{1}^{*}\right)^{2}+C_{1} x_{1}^{*}+C_{5}\right), \\
u^{*}\left(x_{1}^{*}\right)= \pm \frac{\gamma_{p} h_{0}}{\omega_{p} L}\left(\frac{\lambda^{*}}{6 D_{V}^{*}}\left(x_{1}^{*}\right)^{3}+\frac{C_{1}}{2}\left(x_{1}^{*}\right)^{2}+C_{5} x_{1}^{*}+C_{6}\right) .
\end{gathered}
$$

The integration constants are determined from the boundary conditions:

$\underline{\mathrm{CE}-\mathrm{CE}}$ (clamped end $x_{1}^{*}=0$ - clamped end $x_{1}^{*}=1, w^{*}(0)=0, w_{, 1}^{*}(0)=0, w^{*}(1)=0, w_{, 1}^{*}(1)=0, u^{*}(0)=0, u^{*}(1)=0$,

$$
\begin{gathered}
\left.C_{3}=0, C_{4}=0, \begin{array}{c}
\frac{C_{1}}{2} L+C_{2}=-\frac{\lambda^{*}}{6 D_{V}^{*}} \\
\frac{C_{1}}{6} L+\frac{C_{2}}{2}=-\frac{\lambda^{*}}{24 D_{V}^{*}}
\end{array}\right\} \Rightarrow C_{1}=-\frac{\lambda^{*}}{2 D_{V}^{*}}, C_{2}=\frac{\lambda^{*}}{12 D_{V}^{*}}, \\
C_{6}=0, C_{5}=\frac{\lambda^{*}}{12 D_{V}^{*}} .
\end{gathered}
$$

After substituting the integration constants (A.8-A.9) into the general solution (A.2)-(A.7), one can obtain:

$$
w^{*}\left(x_{1}^{*}\right)=\frac{\lambda^{*}}{24 D_{V}^{*}}\left(x_{1}^{*}\right)^{2}\left(x_{1}^{*}-1\right)^{2},
$$




$$
u^{*}\left(x_{1}^{*}\right)= \pm \frac{\gamma_{p} h_{0}}{\omega_{p} L} \frac{\lambda^{*}}{6 D_{V}^{*}} x_{1}^{*}\left(x_{1}^{*}-1\right)\left(x_{1}^{*}-\frac{1}{2}\right)
$$

$\underline{\mathrm{CE}-\mathrm{FE}}$ (clamped end $x_{1}^{*}=0-$ free end $x_{1}^{*}=1, w^{*}(0)=0, w_{11}^{*}(0)=0, M_{2}^{*}(1)=0, T_{31}^{*}(1)=0, u^{*}(0)=0, T_{11}^{*}(1)=0$,

$$
\begin{gathered}
\left.C_{3}=0, C_{4}=0, \begin{array}{r}
C_{1}+C_{2}=-\frac{\lambda^{*}}{2 D_{V}^{*}} \\
C_{1}=-\frac{\lambda^{*}}{D_{V}^{*}}
\end{array}\right\} \Rightarrow C_{2}=\frac{\lambda^{*}}{2 D_{V}^{*}}, \\
C_{6}=0, \quad C_{5}=\frac{\lambda^{*}}{2 D_{V}^{*}}, \\
w^{*}\left(x_{1}^{*}\right)=\frac{\lambda^{*}}{24 D_{V}^{*}}\left(x_{1}^{*}\right)^{2}\left(\left(x_{1}^{*}\right)^{2}-4 x_{1}^{*}+6\right), \\
u^{*}\left(x_{1}^{*}\right)= \pm \frac{\gamma_{p} h_{0}}{\omega_{p} L} \frac{\lambda^{*}}{6 D_{V}^{*}}\left[\left(x_{1}^{*}-1\right)^{3}+1\right] .
\end{gathered}
$$

$\underline{\mathrm{CE}-\mathrm{SSE}}$ (clamped end $x_{1}^{*}=0,-\operatorname{simply}$ supported end $\left.x_{1}^{*}=1\right): w^{*}(0)=0, w_{, 1}^{*}(0)=0, w^{*}(1)=0, M_{2}^{*}(1)=0, u^{*}(0)=0, T_{11}^{*}(1)=0$,

$$
\begin{gathered}
\left.C_{3}=0, C_{4}=0, \begin{array}{c}
C_{1}+C_{2}=-\frac{\lambda^{*}}{2 D_{V}^{*}} \\
\frac{C_{1}}{3}+C_{2}=-\frac{\lambda^{*}}{12 D_{V}^{*}}
\end{array}\right\} \Rightarrow C_{1}=-\frac{5}{8} \frac{\lambda^{*}}{D_{V}^{*}}, C_{2}=\frac{\lambda^{*}}{8 D_{V}^{*}}, \\
C_{6}=0, C_{5}=\frac{\lambda^{*}}{8 D_{V}^{*}}, \\
w^{*}\left(x_{1}^{*}\right)=\frac{\lambda^{*}}{24 D_{V}^{*}}\left(x_{1}^{*}\right)^{2}\left(x_{1}^{*}-1\right)\left(x_{1}^{*}-\frac{3}{2}\right), \\
u^{*}\left(x_{1}^{*}\right)= \pm \frac{\gamma_{p} h_{0}}{\omega_{p} L} \frac{\lambda^{*}}{6 D_{V}^{*}} x_{1}^{*}\left(\left(x_{1}^{*}\right)^{2}-\frac{15}{8} x_{1}^{*}+\frac{6}{8}\right) .
\end{gathered}
$$

\section{Acknowledgements}

The financial support by APVV-0032-10 (Slovak Research and Development Agency) is greatfully acknowledged. 


\section{REFERENCES}

Atluri, S. (2004) The Meshless Method (MLPG) for Domain \& BIE Discretization. Forsyth: Tech Science Press.

Benveniste, Y. (1987) A new approach to the application of MoriTanaka's theory in composite materials. Mechanics of Materials, 6, pp.147-157.

Chamis, C. C. - Sendeckyj, G. P. (1968) Critique on theories predicting thermoelastic properties of fibrous composites. Journal of Composite Materials, 2, 3, pp. 332-358.

Gibson, R. F. (1994) Principles of composite material mechanics. McGraw-Hill.

Hashin, Z. - Rosen, B. W. (1964) The elastic moduli of fiber-reinforced materials. ASME Journal of Applied Mechanics, 4, pp. 223-232.

Hashin, Z. (1979) Analysis of properties of fiber composites with anisotropic constituents. ASME Journal of Applied Mechanics, 46, pp. 543-550.

Koizumi, M. (1993) The concept of FGM. Ceramic Transactions. Functionally Gradient Materials 34, pp. 3-10.
Lancaster, P. - Salkuskas, K. (1981) Surfaces generated by moving least square method. Mathematics of Computation, Vol. 37, pp. 141-158.

Mori, T. - Tanaka, T. (1973) Average stress in matrix and average elastic energy of materials with misfitting inclusions. Acta Metallurgica, Vol. 21, pp. 571-574.

Sladek, V. - Sladek, J. - Zhang, Ch. (2008) Computation of stresses in non-homogeneous elastic solids by local integral equation method: a comparative study. Computational Mechanics, Vol. 41, pp. $827-845$.

Sladek, V. - Sladek, J. - Sator, L. (2013) Physical decomposition of thin plate bending problems and their solution by mesh-free methods. Engineering Analysis with Boundary Elements, Vol. 37, pp. 348-365.

Suresh, S. - Mortensen A. (1998) Fundamentals of Functionally Graded Materials. Institute of Materials, London.

Yamanouchi, M. - Koizumi, M. - Hirai, T. - Shiota, I. (eds.) (1990) FGM-90, Proceedings of the First International Symposium on Functionally Graded Materials. Sendai, Japan, FGM Forum, Tokyo. 